\title{
A FREQUENCY-AGILE INTERFEROMETER FOR SOLAR MICROWAVE SPECTROSCOPY
}

\author{
G. J. HURFORD, R. B. READ, and H. ZIRIN \\ Solar Astronomy 264-33, California Institute of Technology, Pasadena, CA 91125, U.S.A.
}

(Received 10 July, 1984)

\begin{abstract}
A high-resolution microwave spectrometer has been developed by converting the Owens Valley solar interferometer to frequency-agile operation. The system uses $27 \mathrm{~m}$ antennas equipped with phase-locked receivers which can change their observing frequency in 25 or $50 \mathrm{~ms}$. Microwave spectra between 1 and $18 \mathrm{GHz}$ are obtained in a few seconds by successive observations at up to 86 discrete frequencies. At each frequency the data are equivalent to the total power from each antenna and the interferometric amplitude and phase. All data are fully calibrated with respect to cosmic sources.

The instrument was motivated by the need for better microwave spectral resolution for the study of plasma parameters, non-thermal electrons and coronal magnetic field strengths in solar flares and active regions. Early observations with the system are illustrated by a sequence of flare spectra featuring cases with exceptionally narrow continuum bandwidths.
\end{abstract}

\section{Introduction}

Microwave emission has been a relatively neglected domain of solar spectroscopy. Most microwave spectra have been acquired by comparison of independent, single-frequency observations, often made with separate telescopes. Typically, such measurements have been limited to one data point every octave for a frequency-resolution $\mathrm{d} v / v \sim 1$ (e.g., Castelli and Guidice, 1976).

There are a few exceptions to this pattern. High spectral resolution measurements have been reported by Soviet workers using the RATAN-600 telescope between 5 and $7 \mathrm{GHz}$ (Kaverin et al., 1979). At lower frequencies the dynamic spectra of metric and decimetric bursts have been observed with high spectral and time resolution by many instruments (Krüger, 1979). The acquisition of such data on film, however, has made the intensity calibration difficult. Although this situation is changing with the development of digital acquisition systems to provide calibrated spectral data up to $1 \mathrm{GHz}$ (Perrenoud, 1982), at microwave frequencies there has been an almost total absence of high spectral resolution solar data, particularly with polarization and spatial resolution.

This gap in our observational view of the sun is unfortunate for the microwave spectrum contains important information, especially for studies of solar activity. For example, in active regions, interferometric observations at single frequencies often show compact, optically thick sources with coronal brightness temperatures (for reviews, see Schmahl, 1980; Marsh and Hurford, 1982). Such sources are due to gyroresonance opacity which is effective at frequencies that are low multiples of the local gyrofrequency. The gyrofrequency is, of course, determined solely by the coronal magnetic field. To fully exploit such effects in a potential coronal magnetograph, for example, requires sufficient spectral resolution $(\sim 10 \%)$ to distinguish the gyroresonant harmonics. Corresponding 
insights for non-thermal emission can be obtained from microwave spectroscopy of impulsive bursts (e.g. Dulk and Marsh, 1982; Dulk and Dennis, 1982).

Motivated by such considerations, the solar interferometer at the Owens Valley Radio Observatory (OVRO) has been converted to frequency-agile operation with the objective of conducting interferometric microwave spectroscopy between 1 and $18 \mathrm{GHz}$. The purpose of this paper is to describe the system developed for such measurements. In Section 2, an overview of the interferometer is given with the frequency-agile receivers described in more detail in Section 3. Calibration and data processing are discussed in Section 4. Section 5 presents a sequence of flare spectra, summarizes the development of the interferometer, and outlines future plans.

\section{Overview of the Frequency-Agile Interferometer}

The solar interferometer is based on the $27 \mathrm{~m}$ antennas of the OVRO centimetric interferometer, originally developed in 1959 primarily for sidereal sources. In 1976 new receivers and associated hardware were added (Zirin et al., 1978) to meet the distinct requirements of solar observations. In 1979 the time resolution was upgraded from $1 \mathrm{~s}$ to provide a choice of 25,50 , or $100 \mathrm{~ms}$ integration times. In this form, the system provided high time resolution interferometric data at $10.6 \mathrm{GHz}$ during the Solar Maximum Year.

The conversion of the system from a fixed-frequency interferometer to a microwave spectrometer is based on the concept of frequency-agility. This makes use of receivers that can be returned very rapidly over a wide range of frequencies ( 1 to $18 \mathrm{GHz}$ ). Observations are made in quick succession at different frequencies. If this can be done on a sufficiently short timescale that the incident spectrum does not change appreciably, then the solar microwave spectrum can be studied with a single, frequency-agile instrument.

The three major components of the interferometer are the antennas, the receivers, and the backend signal processing hardware. Their operation is controlled and coordinated by a PDP-11/34 minicomputer. Figure 1 provides an overview of the system for the case of the two-element interferometer.

The antennas are fully steerable, $27 \mathrm{~m}$ diameter paraboloids $(f / d=0.4)$, with equatorial mounts providing hour angle coverage of $\pm 4 \mathrm{hr}$ (slightly less for declinations below - 20 degrees). Antenna pointing is controlled by the computer which compares the desired antenna position with the current hour angle and declination (as indicated by 17-bit encoders), and then issues appropriate angular velocity commands. Typical tracking jitter (exclusive of systematic pointing errors) is $\sim 0.1$ arc min. The moveable antennas can be located at any of 17 stations (Figure 2) to provide baselines up to $0.67 \mathrm{~km}$ east-west or $0.49 \mathrm{~km}$ north-south. For three element interferometry, the OVRO $40-\mathrm{m}$ antenna can be included. This provides baselines up to $1.25 \mathrm{~km}$ east-west which correspond to a resolution (fringe spacing) of $2.7 \mathrm{arcsec}$ at $18 \mathrm{GHz}$.

Receivers, discussed in Section 3, are mounted at the prime focus of each antenna. 


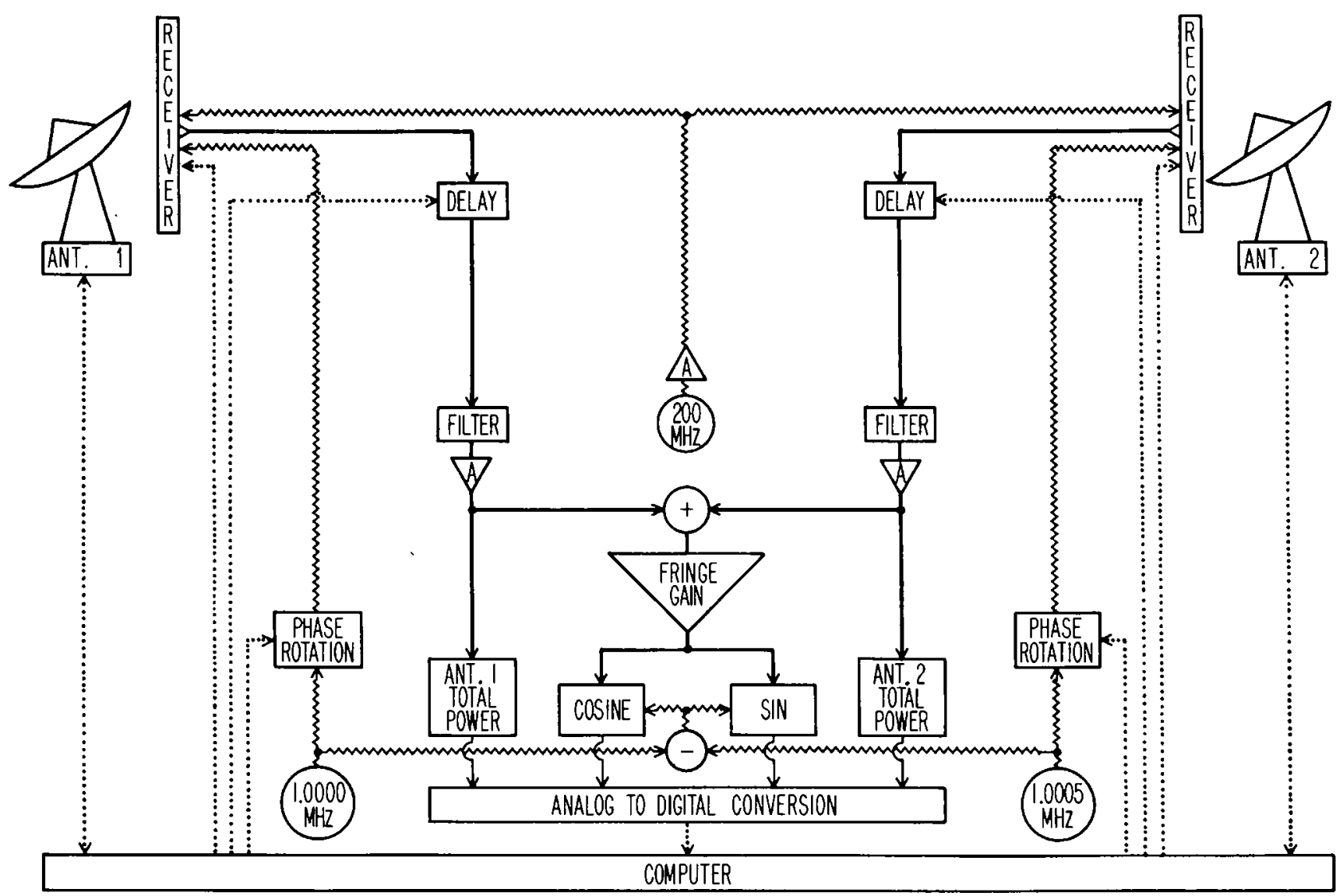

Fig. 1. Simplified schematic of the two-element frequency-agile interferometer. Different types of control and data signals are indicated as follows: . digital; mmanm $\mathrm{CW}$ (single frequency); IF; quasi-DC voltages.

$\otimes$

8

$\otimes$

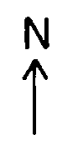

$\otimes$

$$
100 \mathrm{~m}
$$

$\otimes \otimes \stackrel{C \otimes}{\otimes} \otimes \otimes \otimes \quad \otimes \quad \otimes \quad \otimes$

Fig. 2. Interferometer configuration, showing the possible locations of the $27 \mathrm{~m}$ antennas $(x)$ relative to the $40 \mathrm{~m}$ telescope $(\mathrm{T})$ and the control room (C). 
to $18 \mathrm{GHz}$ ) to radiation whose sense of circular polarization matches the sense of the spiral. To measure both right and left circular polarization, a pair of oppositely-polarized feeds are used. They are mounted on a plate that can be rotated like a lens turret to precisely interchange each feed between two positions, one of which is at the prime focus. A coaxial switch selects the feed with the desired polarization as the source of the microwave signal. This signal then passes through a rotary joint to the remainder of the receiver. At this point a noise source with output over the range 1 to $18 \mathrm{GHz}$ can be activated to inject a reproducible signal increment. This can be used to provide a secondary calibration of system gain.

The signal (which still includes 'RF' signals from 0.5 to $18 \mathrm{GHz}$ ) then goes to one input of a mixer/preamplifier. The other input, which determines the observing frequency, is generated by a local oscillator (LO) in the receiver. The mixer functions as a high-frequency switch operating at the LO frequency. The mixer output, therefore, includes the sum and difference of the LO frequency and incident spectrum. Using a low-pass filter to discard any mixer outputs above $60 \mathrm{MHz}$, we are left with a signal (the IF signal) which coherently represents the incident radiation within $\pm 60 \mathrm{MHz}$ of the LO frequency.

For solar applications, a potentially wide range of IF signal levels must be accomodated. This is done by passing the amplified IF signal through a set of attenuators. These are controlled by the computer which inspects the data in real time and attenuates the signals accordingly in steps of $5 \mathrm{db}$ over a range of 0 to $55 \mathrm{db}$. (These levels are restored during data reduction.) Combined with the capabilities of the back-end electronics, this provides the system with an overall dynamic range of $10^{9}$ (nominally $0.4 \mathrm{Jy}$ to $4 \times 10^{4} \mathrm{sfu}$ ). This accommodates both cosmic sources and the full range of solar signal levels expected as a function of observing frequency and solar activity.

As we have seen, the observing frequency is determined by the local oscillator. To cover the range, 1 to $18 \mathrm{GHz}$, three such oscillators $(1-2.2,2-8$, and $8-18 \mathrm{GHz})$ are needed. A pair of switches chooses which of the three oscillator outputs is used at any one time. Each oscillator is a solid state 'YIG-tuned' device whose output frequency is linearly related to an input 'tuning' current. In this application the tuning current (and hence the frequency) is specified by the computer in terms of a 12-bit integer.

This procedure determines the observing frequency to better than $5 \mathrm{MHz}$. For interferometry, however, the LO output in separate receivers must maintain a constant phase relationship to each other. (That is, their observing frequencies must be the same to within $\sim 10^{-4} \mathrm{~Hz}$ ). To accomplish this, each receiver is 'phase-locked' to a common reference as follows. A $200 \mathrm{MHz}$ signal is generated in the control room, amplified, split, and sent to the two receivers (Figure 1). In each receiver, this reference signal provides the input to a harmonic generator whose output includes all the harmonics of $200 \mathrm{MHz}$, over the range 1 to $18 \mathrm{GHz}$. This set of harmonics and the $\mathrm{LO}$ output are the inputs to a mixer whose output spectrum includes that frequency which is the difference of the LO frequency and the nearby harmonic of $200 \mathrm{MHz}$. A phase-lock circuit fine-tunes the LO frequency until this difference is precisely matched to a $1 \mathrm{MHz}$ reference signal which has also been sent from the control room. In this way the observing frequency 


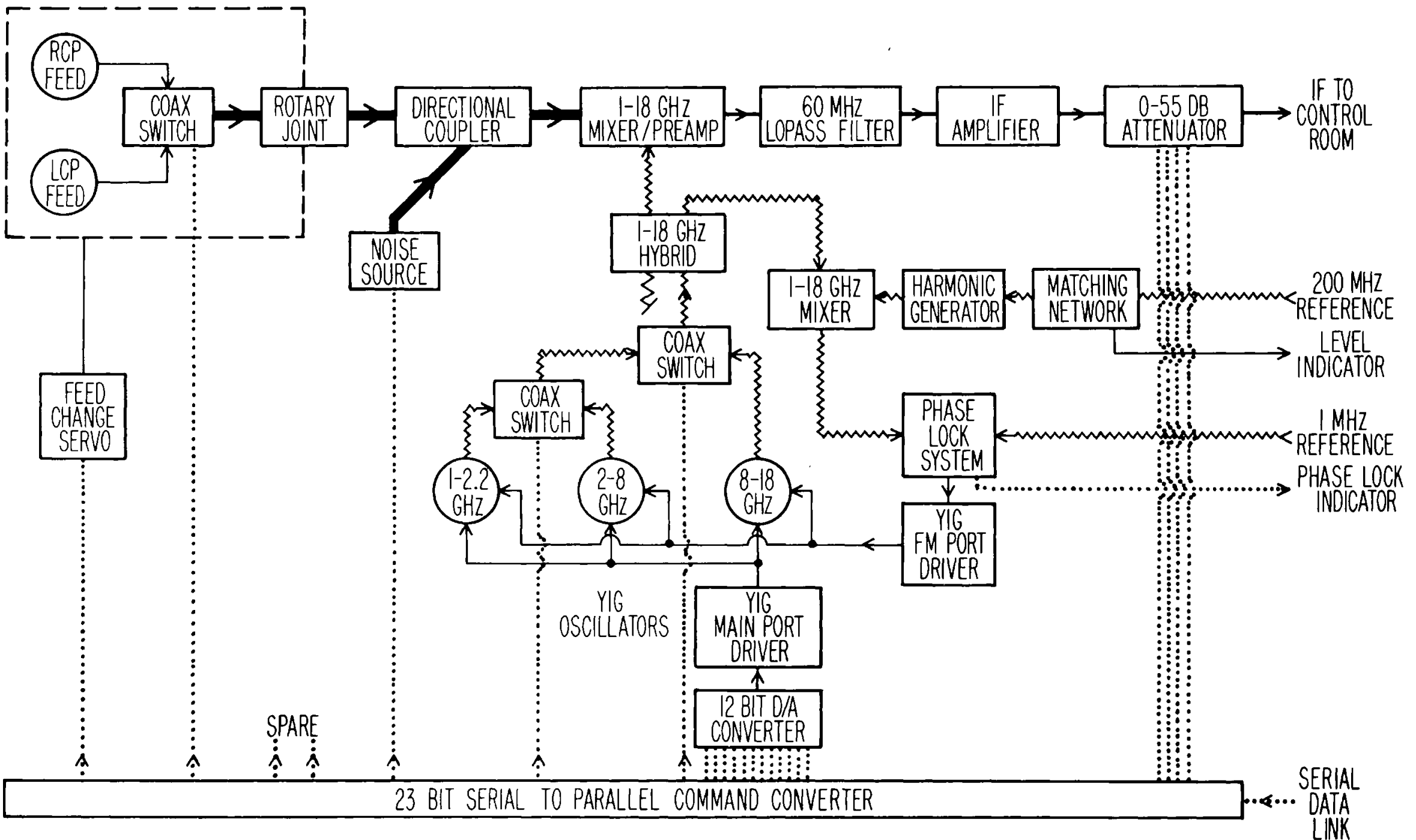


Their purpose is to convert a small, specific band of frequencies in the focussed microwave radiation to the form of amplified, lower 'intermediate frequency' (IF) signals for transmission through cables to the control room. For interferometry, the receiver outputs from different antennas are combined. For this process to be efficient, the separate IF inputs to the correlator or summing circuit must correspond to the same incident wavefront. (The optical analogy here would be to white-light fringes.) In this case, the compensation for the different (air and cable) paths taken by the signals received at each antenna is achieved by sending the IF signals through variable delay lines. On reaching the control room they are then filtered, amplified, summed, and detected in the back-end signal processing system shown in Figure 1.

Rather than use a conventional correlator for combining the signals from the pair of antennas, the signals are added and then detected by a single square-law detector. The observing frequencies of the two receivers are deliberately offset by $500 \mathrm{~Hz}$ with respect to each other. For the correlated component of the incident radiation, this modulates the detector output with a period of $500 \mathrm{~Hz}$. These 'fringes' can then be selectively amplified as needed.

Each measurement represents an integration over a 25,50 , or $100 \mathrm{~ms}$ interval and includes the total power from each antenna, and the cosine and sine components of the fringes. (A rectangular-to-polar conversion makes the latter two data channels equivalent to the fringe amplitude and phase.) At the end of each interval, the integrators are reset so that successive measurements are completely independent. The digitized data are written to magnetic.tape along with housekeeping information such as frequency, polarization, gain settings, antenna-tracking status, etc.

Typical observations consist of $90 \mathrm{~min}$ of multifrequency solar observations alternating with observations of a cosmic source to provide timely calibration of system phase and gain.

\section{Frequency-Agile Receivers}

In the interferometer system outlined above, it is worth noting that only the antennas and receivers are required to respond from 1 to $18 \mathrm{GHz}$. Since the parabolic antennas are, of course, inherently wideband, it is the receivers (Figure 3) that are the critical element in the system. Viewed as black boxes, their input is the focussed radiation field and their IF output is a phase-coherent representation of a specific narrow range of frequencies of the incident spectrum. Receiver operation is controlled by the computer by means of 23-bit commands sent over serial data links. We now consider their operation in more detail.

At the front of each receiver, a 'feed' is located at the prime focus of the antenna. The role of the feed is to convert the focussed free-space radiation into signals on a coaxial cable. In this case, each feed is a 'cavity-backed planar spiral' which consists of spiral-shaped conductors, etched onto an insulating board which forms the front of an absorber-filled, cylindrically-shaped conducting cavity. Since the logarithmic spiral has no natural size scale, the feed responds uniformly over a wide range of frequencies $(0.5$ 
in each receiver maintains a fixed phase relationship to reference frequencies available to both receivers, and so to each other. The resulting phase-locked observing frequencies are given by $(N \times 200 \mathrm{MHz}-1 \mathrm{MHz})$ where $N$ is an integer between 5 and 90 (the harmonic number).

Frequency changes are initiated by the computer by specifying a new coarse tuning current corresponding to a different harmonic of the $200 \mathrm{MHz}$ reference. Frequency changes which do not involve switching between LO's require $16 \mathrm{~ms}$ to be implemented (including the re-establishment of phase-lock). Selection of a different LO can add another $30 \mathrm{~ms}$. Polarization switching requires $500 \mathrm{~ms}$. The software synchronizes these changes with the data acquisition intervals of 25,50 , or $100 \mathrm{~ms}$. Successive intervals can be devoted either to measurements or to frequency/polarization changes. At present, the system can observe at up to 20 different frequencies per second.

It is worth emphasizing that frequencies are not swept in a continuous manner. Rather the system successively observes at discrete frequencies in a pre-selected but arbitrary order. This provides a great deal of flexibility in observing plans since the sequence and timing of observing frequencies and polarizations can be optimized to trade-off time resolution, frequency-resolution and/or frequency coverage to match an experiment's objective, or modified in real-time to reflect transient solar conditions.

\section{Calibration and Data Processing}

\subsection{Frequency CALibration}

Since the computer is responsible for tuning the LO's to within $\sim 9 \mathrm{MHz}$ of the desired observing frequency, it is necessary to determine the digital equivalent of the coarse tuning current for each receiver at each observing frequency. This frequency calibration is fully automated and makes use of a novel feedback signal from the receiver to the computer. In particular, the phase-lock circuit generates an audio frequency which represents the difference between the coarse frequency set by the computer and the correct frequency setting (as set by the phase-lock process). This audio tone is sent back to the control room and monitored by the computer, which then optimizes the coarse tuning current accordingly. Such calibrations can also be conducted non-intrusively during normal frequency-agile observations so that on a slow time scale the computer plays an active role in the phase-lock process, compensating for thermal drifts, etc. The audio feedback also enables the observer to literally listen to the character of the phase-lock behavior, a capability which has proven quite helpful during the development of the system.

\subsection{Total POWER CALIBRation}

The total power response as a function of frequency is determined by calibration on strong cosmic sources such as Cass A. Such calibrations are conducted by rapidly switching between the on- and off-axis feeds when the on-axis feed views the calibrator source. This 'Dicke switching' minimizes the effects of small drifts in system gain on 
difference measurements. To identify inherent differences between the feeds, the process is repeated when no sources are in the field of view. The difference of the two difference signals can then be attribued to the known flux of the cosmic source. Since this calibration is quite time-consuming (approximately $3 \mathrm{~min}$ per frequency) the internal noise diode source is used as a secondary standard for routine calibration. Over a time scale of months, we find that the relative gain as a function of frequency is stable to within a few percent.

\subsection{Polarization calibration}

Although the calibration procedure outlined above is accurate to better than $\sim 10 \%$ in most cases, this level of precision is not satisfactory for polarization measurements. To obtain more precise polarization calibration, several independent determinations of the relative total power response to the quiet sun were made. Assuming that the average sun is unpolarized, this established the relative gain of the system in right-circular and left-circular polarization. Lunar observations also are used for this purpose.

\subsection{INTERFERENCE}

Since most of the observations occur outside the protected radio-frequency bands, consideration must be given to external interference. Observing programs to evaluate such effects as a function of frequency, time and antenna orientation have been undertaken. The results indicate that total power observations of the sun are not seriously impaired. At the other sensivity extreme, interferometric observations of cosmic sources are affected to some extent at 5 to 10 of the 86 frequencies.

\subsection{INTERFEROMETRIC CALIBRATION}

Stable interferometer parameters which require calibration include the baseline, and the relative phase and correlated gain as a function of frequency and polarization. These calibrations are based on cosmic sources such as $3 \mathrm{C} 84,3 \mathrm{C} 273$, etc.

\subsection{ROUTINE CALIBRATIONS}

While the detailed frequency dependence of the instrumental parameters discussed above is not necessarily a simple function of frequency, the relative frequency dependence appears to be quite stable. This permits calibration during the actual solar observations to be limited to just a few frequencies. This is sufficient to characterize the smooth frequency dependence of thermal and weather-related effects which vary on a time scale of hours. These more frequent calibrations include phase and amplitude calibration on a cosmic source, noise source calibrations to establish overall system gain, and determination of the effective zeroes of the analog signal processing system. These automated calibrations require $\sim 20 \mathrm{~m}$ to execute.

\subsection{DATA REDUCTION}

Data reduction on the Caltech Astronomy VAX computer consists of three basic steps, all of which can be done in a single pass. The first step consists of editing to eliminate 
flagged data samples. Reasons for such flagging include marginal antenna tracking, phase-lock anomalies, saturated data samples or data acquired while a frequency or polarization change was in progress. Next, the data are calibrated on the basis of frequency-dependent gain tables, adjusted to reflect the results of the routine calibrations. Periodic variations in the phase data (natural fringes), an expected consequence of the Earth's rotation, are compensated at this point. Finally the data at each frequency and polarization are averaged or interpolated to a common time so that the result is the best estimate of the instantaneous spectrum of a changing signal. (In general, we have found that the derived spectra are not sensitive to the interpolation technique.) At this stage, the data have been reduced to the form of a calibrated matrix whose axes represent time and frequency/polarization respectively. Such matrices are, in effect, calibrated dynamic spectra which can then be further manipulated, or displayed as light curves, spectra, etc.

\subsection{SQUINT MODULATION}

We have found a systematic frequency-dependence to the effective centre of the spiral feeds used in the prime focus of the antennas. This results in a corresponding frequencydependence to the telescope pointing, amounting to about $\pm 6 \%$ of the half-powerbeam-width. While this is unimportant for on-axis sources, it can noticeably modulate the polarization spectrum of sources (such as flares) which are located well away from the centre of the primary beam. The frequency-dependence of the pointing has been calibrated, however, and a two parameter fit to the spectra enables most of this effect to be eliminated. Since the parameterization depends on the position of the source, such fits can be exploited to locate the source within the field of view (Hurford, 1983).

\subsection{Spectral SMOOTHING}

Evaluation of the spectra determined by the techniques outlined above must deal with the following consideration. The current precision of $\sim 10 \%$ for the intensity calibration of individual frequencies would be generally satisfactory for spectral analysis if the frequency resolution were low. However for frequency resolution significantly better than $10 \%$, this level of precision causes the resulting spectra to appear unnecessarily 'noisy' at the $10 \%$ level. Since this complicates the evaluation of spectral features, we have found it sometimes useful to smooth the resulting spectra. (An algorithm is used which makes no a priori assumptions as to the spectral form or shape and which does not systematically affect either the average level or slopes of the spectra.)

Finally, it should be emphasized that as of this writing the calibration of the interferometer is an ongoing process. Considerable improvement in both precision and allowance for systematic effects is anticipated as observations and calibrations continue and as appropriate improvements are made. 


\section{Development Status and Initial Results}

\subsection{EARLY obSERVATIONS}

The first frequency-agile receiver became operational in April 1981. This receiver differed slightly from those described above in that it contained a fourth local oscillator, phase-locked at $10.6 \mathrm{GHz}$, in addition to the three YIG-tuned oscillators covering the range 1 to $18 \mathrm{GHz}$ which were not phase-locked at that time. Until mid-1982, this receiver provided high spectral resolution data in total power; a fixed-frequency receiver on the other antenna observed with high time resolution $(50 \mathrm{~ms})$ at $10.6 \mathrm{GHz}$; and the combination was used intermittently as an interferometer at $10.6 \mathrm{GHz}$ every few seconds. Extensive solar observations in this 'hybrid' mode between May and October 1981 provided high spectral resolution data for many flares.

\subsection{Flare spectra}

To illustrate such flare data, Figures 4 and 5 show a small fraction of the light curves and total power spectra obtained for a burst that occured on 1981, August 7.

The light curves at 2 and $8 \mathrm{GHz}$ show the impulsive phase of the event beginning at 19:00 UT. At the intermediate frequency of $4 \mathrm{GHz}$, however, microwave emission began 4 min earlier. Reference to the sequence of spectra in Figure 5 shows that the pre-impulsive emission (before 19:00 UT) had a relatively narrow bandwidth and sharp high and low frequency cutoffs. In this case the full-width-half-power was $\sim 60 \%$ of the peak frequency.

Such narrow-bandwidth spectra would have been difficult to study with previous patrol data. We sometimes see them as isolated events in conjunction with large bursts as in the case illustrated. Their narrow spectral widths suggest that they originate in a region of relatively uniform physical conditions.

The large value of the low-side spectral index (as high as $\sim 6$ in the case illustrated) has implications for the physical origin of the low frequency cutoff. While optically thick thermal sources can have a maximum spectral index of 2, and gyrosynchrotron self-absorption can explain low frequency spectral indices of up to 3 (Dulk and Marsh, 1982), steeper spectra require a different mechanism, such as external gyroresonance or free-free absorption (Melrose, 1980), or Razin suppression.

\subsection{Present status}

Observations with the frequency-agile interferometer in the configuration described in Sections 3 and 4 began in October 1982 and an extensive program of flare and active region observations has been undertaken since. The use of a two-element interferometer has several advantages over a single-antenna for microwave spectroscopy. First, it suppresses signals from the extended, background sun to permit the interferometer to isolate the signals from compact sources of the nonflaring active region. Thus the system can be conveniently determine the active region spectrum prior to and following flare activity. Second, it also provides significant practical gain in sensitivity to extend the 


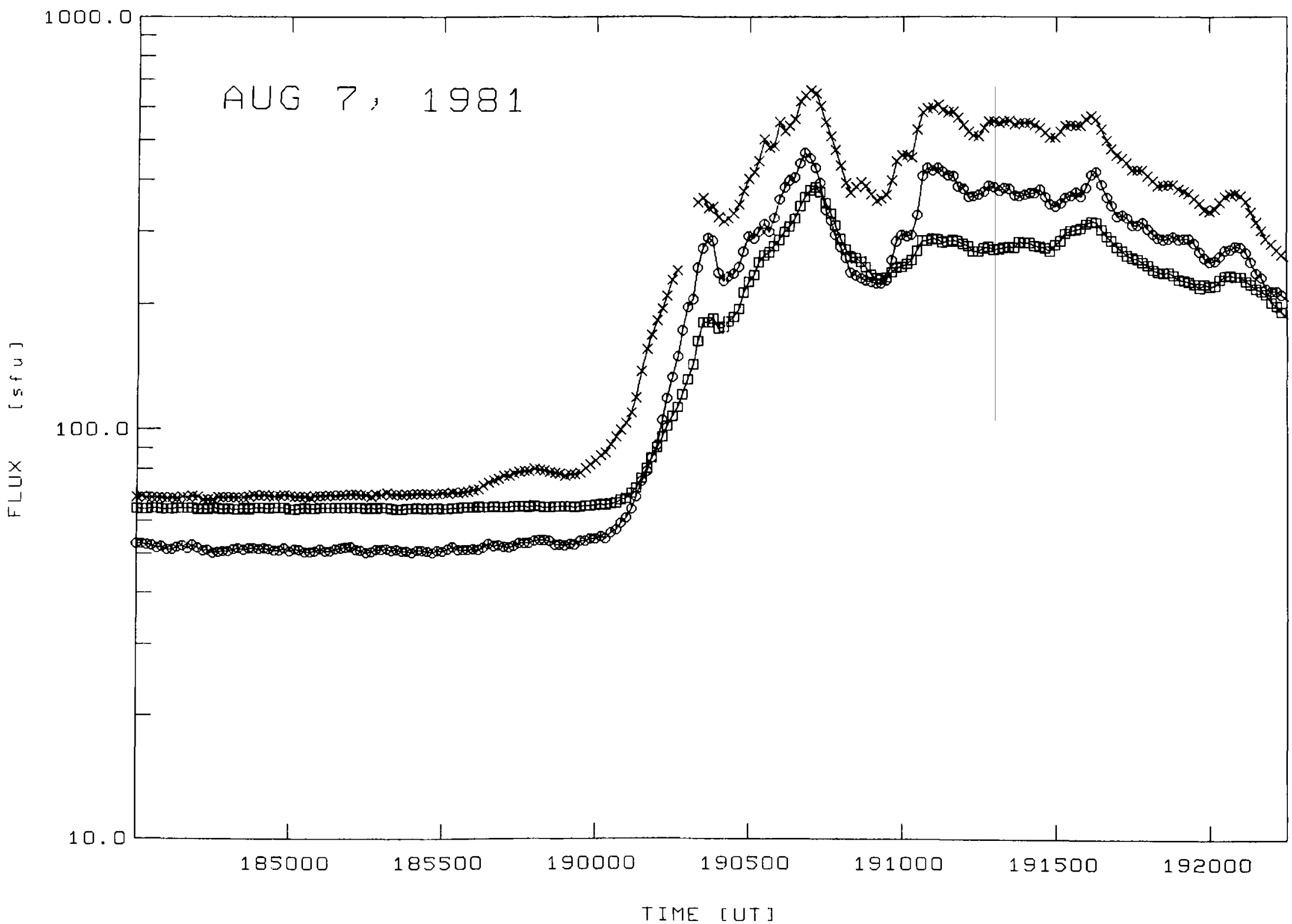

Fig. 4. Light curves for a class M3.9, Importance 2B flare on 1981, August 7 at $2 \mathrm{GHz}(\square), 4 \mathrm{GHz}(\times)$, and $8 \mathrm{GHz}(O)$. Note that the preimpulsive phase emission before 19:00 UT was at the intermediate frequency of $4 \mathrm{GHz}$. 


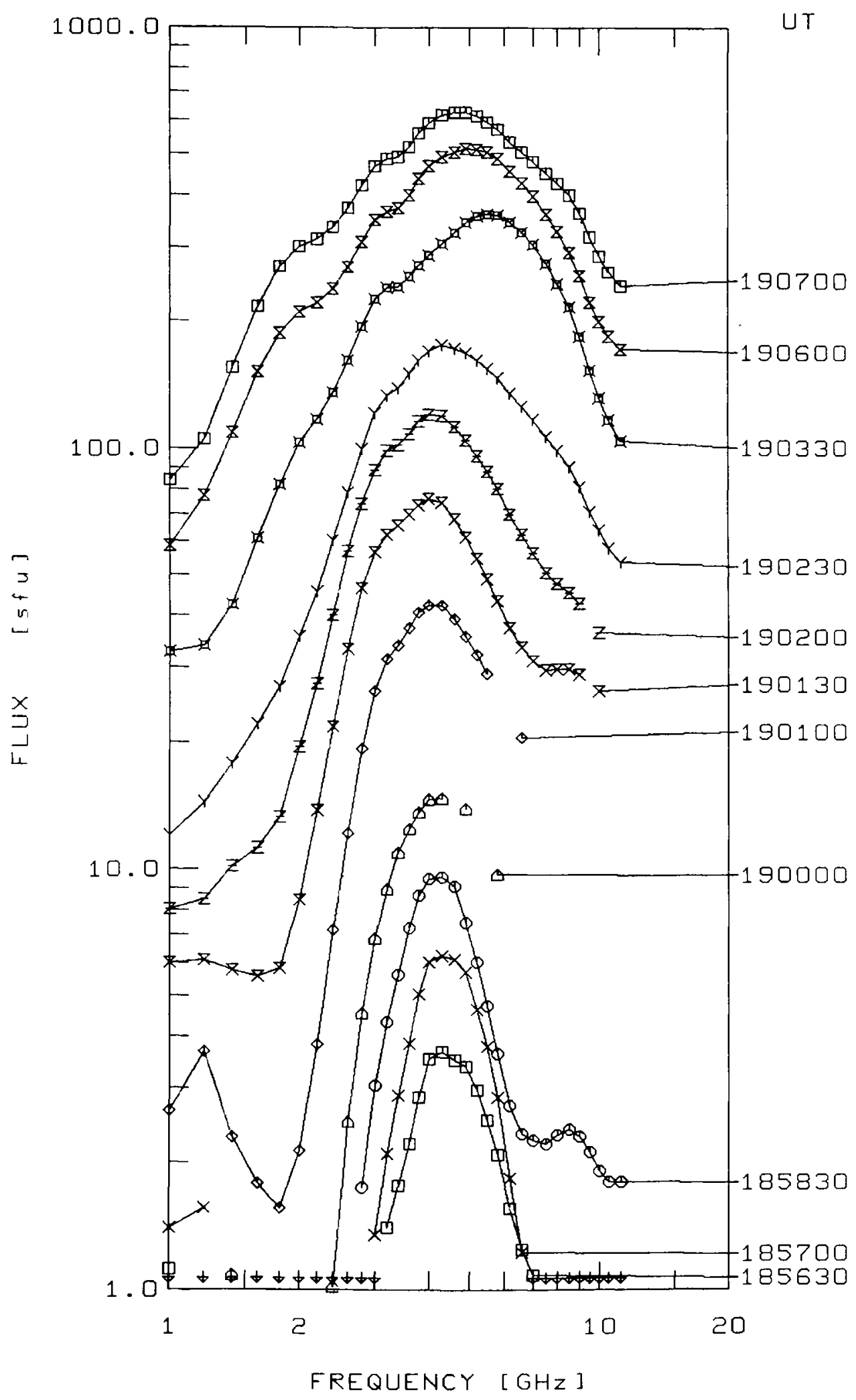

Fig. 5. Sequence of microwave spectra for the early stages of the event shown in Figure 4. Preflare levels have been subtracted at each frequency. Times are indicated at right. 
spectrum of small bursts to lower levels than is possible with total power data. Third, it provides information on the source location.

The combination of frequency-agility and interferometry is significant since the separation of the antennas in units of wavelength is different at each frequency. Thus, interferometric measurements as a function of frequency sample different spatial Fourier components of the source, (i.e. different points in the $u-v$ plane). If the source is unresolved, this does not complicate the spectral analysis. If the source is partially resolved and the spectrum partially understood, this provides a potentially useful imaging capability. Although the practicality of such imaging remains to be explored, at a minimum it will permit the removal of lobe ambiguities normally associated with a two-element interferometer.

Interferometric results will be the subject of subsequent papers.

\subsection{Future PLANS}

Apart from incremental hardware/software improvements and expected refinements in calibration, the next major step in solar spectroscopy at OVRO will be the use of a third frequency-agile receiver, similar in design to the two already in service. This third receiver will be used on an occasional basis on the $40 \mathrm{~m}$ antenna in conjunction with the present system to permit three-element frequency-agile interferometry. The resulting set of three baselines at each frequency will add two significant capabilities. First, it will enable snapshot imaging in two dimensions, rather than one. Second, it will permit the determination of source size at each frequency and polarization. Since at each frequency, the brightness temperature is determined by the ratio of source flux to area, this will enable the brightness temperature spectrum of solar bursts to be measured for the first time. This is important for it is the brightness temperature spectrum (as distinct from the flux spectrum) that is derived from most theoretical models. Therefore we are hopeful that this development will provide new scope for the effective interaction of theory and observation in the study of solar flares.

\section{Acknowledgements}

We gratefully acknowledge the contributions of Rich Goeden, David Thompson, and Simon Groetz to the development of this system and appreciate the assistance of the staff at OVRO, most particularly Dan Daugherty and Chuck Spencer.

Receiver fabrication was funded by NSF Grants AST-8018200 and ATM-8207281. Support for solar observations at OVRO was provided by the NSF Grants AST-7916149 and AST-8115969. Data analysis was funded by AFGL Contract F19628-82-K-0033 and NSF Grants ATM-8108449 and ATM-8309955. We are grateful to Dr R. E. Vogt for his support and for his assistance in arranging funding for the third receiver. 


\section{References}

Castelli, J. P. and Guidice, D. A.: 1976, Vistas Astron. 19, 355.

Dulk, G. A. and Dennis, B. R.: 1982, Astrophys. J. 260, 875.

Dulk, G. A. and Marsh, K. A.: 1982, Astrophys. J. 259, 350.

Hurford, G. J.: 1983, Bull. Am. Astron. Soc. 15, 973.

Kaverin, N. S., Kobrin, M. M., Korshunov, A. I., and Shushunov, V. V.: 1979, Solar Phys. $63,379$.

Kruger, A.: 1979, Introduction to Solar Radio Astronomy and Radio Physics, D. Reidel Publ. Co., Dordrecht, Holland.

Marsh, K. A. and Hurford, G. J.: 1982, Ann. Rev. Astron. Astrophys. 20, 497.

Melrose, D. B.: 1980, Plasma Astrophysics: Nonthermal Processes in Diffuse Magnetized Plasmas, Vol. 1, Gordon and Breach, New York.

Perrenoud, M. R.: 1982, Solar Phys. 81, 197.

Schmahl, E.: 1980, in M. R. Kundu and T. E. Gergely (eds.), 'Radio Physics of the Sun', IAU Symp. 86, 71.

Zirin, H., Hurford, G. J., and Marsh, K. A.: 1978, Astrophys. J. 224, 1043. 